Теорія Ймовір. та Матем. Статист. Вип. 76, 2007
Theor. Probability and Math. Statist. No. 76, 2008, Pages 155-158 S 0094-9000(08)00739-4

Article electronically published on July 16, 2008

\title{
LIMIT THEOREM FOR MAXIMAL SEGMENTAL SCORE FOR RANDOM SEQUENCES OF RANDOM LENGTH
}

UDC 519.21

\section{B. L. S. PRAKASA RAO AND M. SREEHARI}

\begin{abstract}
We obtain the limiting distribution of the maximal segmental score for the partial sums for a random number of independent and identically distributed random variables.
\end{abstract}

\section{INTRODUCTION}

Sequence alignment is useful for discovering functional, structural and evolutionary information in biological sequences. If two sequences from different organisms are similar, it could be because the organisms had a common ancestor sequence which transformed into these two organisms due to evolutionary changes (mutation). Alignment refers to the procedure of comparing two or more biological sequences by looking for a series of individual characters or character patterns that are in the same order in the sequences. A major problem is to identify interesting patterns in sequences. Suppose $A_{1}, \ldots, A_{n}$ is an observed sequence (DNA, protein, etc.) from a finite alphabet (nucleotides or amino acids). Let $\sigma$ be a scoring function. The local score of the sequence $A_{1}, \ldots, A_{n}$ according to the scoring scheme $\sigma$ is defined by

$$
H_{n}=\max _{1 \leq i \leq j \leq n}\left(\sum_{k=i}^{j} \sigma\left(A_{k}\right)\right) .
$$

The local score $H_{n}$ corresponds to a segment of the sequence with maximal aggregate score. The properties of the local score $H_{n}$ have been investigated using the probability model that the successive letters of a sequence are generated by independent and identically distributed (i.i.d.) random variables or with a Markov chain model. Arratia and Waterman [2] proved that, for a sequence of length $n$, as $n \rightarrow \infty$, there exists a transition phase; when the average score is positive, there is a linear growth of the local score in $n, H_{n}=O(n)$; and when the average score is negative, it is called the logarithmic case, $H_{n}=O(\log n)$. Arratia et al. [1 investigated the approximation of counts of matches in the best matching segment of specified length when comparing two long sequences of i.i.d. letters. Mercier and Daudin [7] obtain the exact distribution for the local score of one i.i.d. sequence. Mercier et al. [8] established the exact distribution of the maximum partial sum of a sequence of i.i.d. random variables using random walk theory, which led to a new approximation of the distribution of the local score of a sequence.

Let $\left\{X_{n}\right\}$ be a sequence of independent and identically distributed (i.i.d.) random

2000 Mathematics Subject Classification. Primary 60G50.

Key words and phrases. Maximum segmental score, limit theorem, random sequences, random length. 
variables defined on a probability space $(\Omega, \mathcal{F}, \mathrm{P})$ satisfying the following assumptions:

(i) $\mathrm{P}\left(X_{1}>0\right)>0$,

(ii) $\mathrm{E}\left(X_{1}\right)<0$, and

(iii) the random variable $X_{1}$ is bounded; i.e., $\mathrm{P}\left(\left|X_{1}\right|<c\right)=1$ for some constant $c>0$.

Let $S_{0}=0, S_{n}=\sum_{k=1}^{n} X_{k}, n \geq 1$, and $Z_{n}=\max _{1 \leq i \leq j \leq n}\left(S_{j}-S_{i}\right)$. Iglehart [5] proved the following theorem.

Theorem 1.1. If $\left\{X_{n}\right\}$ is a sequence of non-lattice i.i.d. random variables satisfying the conditions given above, then

$$
\mathrm{P}\left[Z_{n}-\theta \log n \leq x\right] \rightarrow G(x)=\exp \left[-k e^{-x / \theta}\right] \text { as } n \rightarrow \infty
$$

for every $x \in \mathbf{R}$, where $\theta$ and $k$ are positive constants depending on the distribution of $X_{1}$.

Karlin and Dembo [6 extended this result to the lattice case. We obtain a random version of Theorem 1.1 in Section 2.

\section{MAin RESUlt}

Let $\left\{N_{n}\right\}$ be a sequence of positive integer-valued random variables defined on the probability space $(\Omega, \mathcal{F}, \mathrm{P})$ satisfying the condition

$$
\frac{N_{n}}{k_{n}} \stackrel{\mathrm{P}}{\rightarrow} N
$$

for some sequence of integers $\left\{k_{n}\right\}, 0<k_{n} \uparrow \infty$, where $N$ is a positive random variable. We prove the following random indexed version of the result in (1.1).

Theorem 2.1. Suppose $\left\{X_{n}\right\}$ is a sequence of non-lattice i.i.d. random variables satisfying the conditions stated above. Then

$$
\mathrm{P}\left(Z_{N_{n}}-\theta \log N_{n} \leq x\right) \rightarrow G(x)
$$

as $n \rightarrow \infty$ for every $x \in \mathbf{R}$, where $G(x)$ is as defined by (1.1), and, for every $x \in \mathbf{R}$,

$$
\mathrm{P}\left(Z_{N_{n}}-\theta \log k_{n} \leq x\right) \rightarrow \int_{0}^{\infty} G(x-\theta \log u) d \mathrm{P}(N \leq u) \quad \text { as } n \rightarrow \infty .
$$

The proof of the theorem depends on the following lemmas.

Lemma 2.2. Let $\left\{r_{n}\right\}$ and $\left\{k_{n}\right\}$ with $r_{n}<k_{n}$ be two increasing sequences of positive integers with $r_{n} \rightarrow \infty$ and let $\left\{A_{n}\right\}$ be a sequence of events such that $A_{n}$ depends only on the random variables $X_{r_{n}}, \ldots, X_{k_{n}}$. Then, for any event $A$ not depending on $n$ such that $\mathrm{P}(A)>0$,

$$
\mathrm{P}\left(A_{n} \mid A\right)-\mathrm{P}\left(A_{n}\right) \rightarrow 0 \quad \text { as } n \rightarrow \infty .
$$

The proof of the result is given in Lemma 1 in Barndorff-Nielsen [3].

Let $r_{n}=o\left(\log k_{n}\right)$, and $Z_{n}^{\star}=\max _{r_{n}+1 \leq i \leq j \leq k_{n}}\left(S_{j}-S_{i}\right)$. Then

$$
Z_{n}^{\star} \triangleq Z_{k_{n}-r_{n}}
$$

in the sense that they have identical distributions, since $X_{1}, X_{2}, \ldots, X_{n}$ are i.i.d. random variables. Hence, as $n \rightarrow \infty$, we have

$$
\mathrm{P}\left(Z_{n}^{\star} \leq x+\theta \log k_{n}\right) \simeq \mathrm{P}\left(Z_{n}^{\star} \leq x+\theta \log \left(k_{n}-r_{n}\right)\right)
$$

because $r_{n}=o\left(\log k_{n}\right)$ in the sense that their difference tends to zero. This can be proved by the following arguments. Let

$$
U_{n}=Z_{n}^{\star}-\theta \log \left(k_{n}-r_{n}\right)
$$


and

$$
F_{n}(x)=\mathrm{P}\left(U_{n} \leq x\right) .
$$

In view of (1.1) and (2.3), it follows that

$$
F_{n}(x) \stackrel{w}{\rightarrow} G(x) \quad \text { as } n \rightarrow \infty .
$$

Note that

$$
\begin{aligned}
\mathrm{P}\left(Z_{n}^{\star} \leq x+\theta \log k_{n}\right) & =\mathrm{P}\left(Z_{n}^{\star} \leq x+\theta \log \left(k_{n}-r_{n}\right)-\theta \log \frac{k_{n}-r_{n}}{k_{n}}\right) \\
& =\mathrm{P}\left(U_{n} \leq x-\theta \log \frac{k_{n}-r_{n}}{k_{n}}\right)=F_{n}\left(x-\theta \log \frac{k_{n}-r_{n}}{k_{n}}\right) .
\end{aligned}
$$

Note that the distribution function $G(x)$ is continuous for all $x$. An application of Polya's theorem and the fact that $r_{n}=o\left(\log k_{n}\right)$ implies that

$$
F_{n}\left(x-\theta \log \frac{k_{n}-r_{n}}{k_{n}}\right) \stackrel{w}{\rightarrow} G(x) \quad \text { as } n \rightarrow \infty .
$$

Hence

$$
\mathrm{P}\left(Z_{n}^{\star} \leq x+\theta \log k_{n}\right) \rightarrow G(x) \quad \text { as } n \rightarrow \infty .
$$

We now establish the Rényi mixing property for the sequence of events $\left\{A_{n}\right\}$, where

$$
A_{n}=\left\{Z_{k_{n}} \leq x+\theta \log k_{n}\right\} .
$$

Lemma 2.3. For $x \in \mathbf{R}$ and $\theta$ as in (1.1) and any event $A$ independent of $n$ such that $\mathrm{P}(A)>0$,

$$
\mathrm{P}\left(A_{n} \mid A\right)-\mathrm{P}\left(A_{n}\right) \rightarrow 0 \quad \text { as } n \rightarrow \infty .
$$

Proof. In view of Lemma 2.2,

$$
\mathrm{P}\left(Z_{n}^{\star} \leq x+\theta \log k_{n} \mid A\right)-\mathrm{P}\left(Z_{n}^{\star} \leq x+\theta \log k_{n}\right) \rightarrow 0 \quad \text { as } n \rightarrow \infty .
$$

The proof of Lemma 2.3 will be complete if we prove that

$$
\mathrm{P}\left(A_{n} \mid A\right)-\mathrm{P}\left(Z_{n}^{\star} \leq x+\theta \log k_{n} \mid A\right) \rightarrow 0 \quad \text { as } n \rightarrow \infty
$$

for any event $A$ with $\mathrm{P}(A)>0$. Let

$$
W_{n}=\max _{1 \leq i \leq r_{n}} \max _{i \leq j \leq k_{n}}\left(S_{j}-S_{i}\right) .
$$

We observe that

$$
A_{n}=\left\{Z_{k_{n}} \leq x+\theta \log k_{n}\right\}=\left\{\max \left(Z_{n}^{\star}, W_{n}\right) \leq x+\theta \log k_{n}\right\}
$$

and hence

$$
\mathrm{P}(A)\left|\mathrm{P}\left(A_{n} \mid A\right)-\mathrm{P}\left(Z_{n}^{\star} \leq x+\theta \log k_{n} \mid A\right)\right| \leq \mathrm{P}\left(Z_{n}^{\star} \leq x+\theta \log k_{n}<W_{n}\right) .
$$

Writing $x+\theta \log k_{n}=a_{n}(x)$, we observe that $W_{n}>a_{n}(x)$ if and only if there exist integers $r$ and $s$ such that $r<s \leq k_{n}, r \leq r_{n}$ for which

$$
\sum_{j=r}^{s} X_{j}>a_{n}(x)
$$

Clearly $s>r_{n}$ because otherwise

$$
\sum_{j=r}^{s} X_{j}<c(s-r+1)
$$


and the inequality (2.7) cannot hold for large $n$. Thus, the event $\left[W_{n}>a_{n}(x)\right]$ implies that for some $r \leq r_{n}<s \leq k_{n}$, the inequality (2.7) holds and for such values of $r$ and $s$,

$$
\begin{aligned}
Z_{n}^{\star} & \geq X_{r_{n}+1}+\cdots+X_{s}=\sum_{j=r}^{s} X_{j}-\sum_{j=r}^{r_{n}} X_{j} \\
& >a_{n}(x)-c\left(r_{n}-r+1\right) \quad\left(\text { because }\left|X_{i}\right|<c\right) \\
& \geq a_{n}(x)-c r_{n} .
\end{aligned}
$$

Thus

$$
\mathrm{P}\left(Z_{n}^{\star} \leq x+\theta \log k_{n}<W_{n}\right) \leq \mathrm{P}\left[a_{n}(x)-c r_{n}<Z_{n}^{\star} \leq a_{n}(x)\right] \rightarrow 0 \quad \text { as } n \rightarrow \infty,
$$

because of the fact at (2.4) and the choice of the sequence $r_{n}$. The proof now follows from (2.6).

The results stated in Theorem 2.1 follow directly from Theorem 1 and Corollary 1 of Csörgö [4] in view of the Rényi mixing property proved in Lemma 2.3.

\section{BIBLIOGRAPHY}

1. R. Arratia, L. Gordon, and M. S. Waterman, The Erdös-Rényi law in distribution, for coin tossing and sequence matching, Ann. Statist. 18 (1990), 539-570. MR.1056326 (92a:60054)

2. R. Arratia and M. S. Waterman, A phase transition for the score in matching random sequences allowing deletions, Ann. Appl. Prob. 4 (1994), 200-225. MR.1258181 (95b:60024)

3. O. Barndorff-Nielsen, On the limit distribution of the maximum of a random number of independent random variables, Acta Math. Acad. Sci. Hungar. 15 (1964), 399-403. MR0170360 (30:598)

4. S. Csörgö, On limit distributions of sequences of random variables with random indices, Acta Math. Acad. Sci. Hungar. 25 (1974), 227-232. MR0365660(51:1912)

5. D. L. Iglehart, Extreme values in the GI/G/1 queue, Ann. Math. Statist. 43 (1972), 627-635. MR $0305498(46: 4628)$

6. S. Karlin and A. Dembo, Limit distributions of maximal segmental score among Markovdependent partial sums, Adv. Appl. Probab. 24 (1992), 113-140. MR.1146522 (93b:60042)

7. S. Mercier and J. J. Daudin, Exact distribution for the local score of one i.i.d. random sequence, J. Comput. Biol. 8 (2001), 373-380.

8. S. Mercier, D. Cellier, and D. Charlot, An improved approximation for assessing the statistical significance of molecular sequence features, J. Appl. Probab. 40 (2003), 427-441. MR1978101 (2004d:60115)

University of Hyderabad, Hyderabad 500046, India

E-mail address: blsprsm@uohyd.ernet.in

M. S. University, VAdodara, India

E-mail address: msreehari03@yahoo.co.uk 